Jean-Jacques Rousseau et les arts visuels, Actes du Colloque de Neuchâtel (20-22 septembre 2001, édités par Frédéric $\mathrm{S}$. Eigeldinger)

\title{
Paola Sosso
}

\section{(2) OpenEdition}

\section{Journals}

Edizione digitale

URL: https://journals.openedition.org/studifrancesi/39457

DOI: $10.4000 /$ studifrancesi.39457

ISSN: 2421-5856

\section{Editore}

Rosenberg \& Sellier

\section{Edizione cartacea}

Data di pubblicazione: 1 décembre 2004

Paginazione: $377-378$

ISSN: 0039-2944

\section{Notizia bibliografica digitale}

Paola Sosso, «Jean-Jacques Rousseau et les arts visuels, Actes du Colloque de Neuchâtel (20-22 septembre 2001, édités par Frédéric S. Eigeldinger)», Studi Francesi [Online], 143 (XLVIII | II) | 2004 online dal 30 novembre 2015, consultato il 19 mai 2021. URL: http://journals.openedition.org/ studifrancesi/39457 ; DOI: https://doi.org/10.4000/studifrancesi.39457

Questo documento è stato generato automaticamente il 19 mai 2021.

\section{cc) (†)}

Studi Francesi è distribuita con Licenza Creative Commons Attribuzione - Non commerciale - Non opere derivate 4.0 Internazionale. 


\title{
Jean-Jacques Rousseau et les arts visuels, Actes du Colloque de Neuchâtel (20-22 septembre 2001, édités par Frédéric S. Eigeldinger)
}

\author{
Paola Sosso
}

\section{NOTIZIA}

Jean-Jacques Rousseau et les arts visuels, Actes du Colloque de Neuchâtel, Faculté des lettres et sciences humaines de l'Université, Association Jean-Jacques Rousseau (20-22 septembre 2001, édités par Frédéric S. EIGELDINGER,) «Annales de la Société Jacques Rosseau», Genève, Droz, 2003, pp. 677.

1 II volume si occupa di un aspetto ancora poco studiato degli scritti del ginevrino, rivalutando l'interesse di Rousseau per le arts visuels sotto svariati punti di vista. Una prima-sezione, dal titolo «Voir», si apre con uno studio ampio e denso nei contenuti di Raymond TRousson (Art et luxe au XVIIIe siècle, pp. 15-36) in cui l'autore colloca la posizione di Jean-Jacques nei confronti del lusso e delle arti all'interno del dibattito settecentesco aperto da Bayle a inizio secolo e portato avanti da esimie personalità dell'epoca: le riflessioni di Rousseau, collocabili sulla scia di una forte reazione contro l'art degradé diffusa verso la metà del secolo, appaiono rivoluzionarie, perché egli, a differenza dei philosophes, è ostile a qualsiasi forma di lusso, è intransigente, e la sua visione del divenire storico esclude ogni compromesso. L'articolo che segue di Richard GLAUSER, dal titolo «De tous nos sens le plus fautif»: infirmité naturelle et expérience de la vue dans l'Emile (pp. 37-58) presenta un interessante paragone tra vista e tatto e studia lo sviluppo della vista nei libri secondo e quarto dell'Emile per concludersi con l'analisi di un significativo confronto operato da Russeau tra vista e arts visuels. Chiude questa prima sezione l'intervento di Frédéric LEFEBVRE dedicato a La société, le spectateur et la 
perspective: histoire d'une métaphore obligée au XVIIIe siècle (pp. 59-78). La seconda sezione è intitolata "Décors et perspectìves»: le riflessioni di Juan CALATRAVA (Rousseau et l'architecture, pp. 81-111), che aprono questa parte dell'opera, prendono spunto dalla constatazione che Jean-Jacques non mostra nelle sue opere un interesse specifico per l'architettura. Questa non assume infatti nei suoi testi un valore estetico, ma diviene tuttavia terreno privilegiato in cui prendono forma idee morali, economiche e politiche, come mostrano, tra gli altri, alcuni brani del primo Discours, la Lettre à d'Alembert, molte pagine dei testi autobiografici e La Nouvelle Héloïse. L 'Elysée: leçon de morale dans un parce (pp. 113-129) è il titolo dello studio di Geneviève GOUBIER-ROBERT in cui l'autrice si interroga sulla posizione di Rousseau nel dibattito attorno al jardin paysagiste. In Décor et pantomimes du Devin du village: une étude didascalique (pp. 131-165) Jacqueline WAEBER risale alle fonti dell'opera (1- «livret autographe», 2- «partition d'orchestre non autographe», 3- «édition gravée de la musique, préfacée par Rousseau») per definire in modo più chiaro alcuni aspetti dell'universo scenico del Devin du village relativi al décor e all'utilizzo della pantomima. Ancora dedicato alle opere meno studiate di Rousseau è 1'articolo di Alain Cernuschi (La dramaturgie du regard dans Narcisse et Pygmalion ou les pièges de l'image émouvante, pp. 167-180), mentre l'intervento che chiude questa seconda sezione, di Maria Costanza PERES PISSARRA (Rousseau, la fête collective et le théâtre, pp. 181-199) analizza la differenza tra una forma di teatro negativa ed una positiva, intesa, quest'ultima, come comunione in cui ciascuno è al contempo attore e spettatore.

La terza sezione, dal titolo «De la gravure et de la peinture», si apre con L'atelier de l'iconoclaste (pp. 203-235), studio in cui Jean STAROBINSKI analizza nei dettagli il ruolo, o meglio i ruoli svolti dalle immagini nelle opere del ginevrino. Ecco due brevi esempi: il primato accordato alla persona di Jean-Jacques relega in molti casi l'immagine a un ruolo subalterno o marginale (è il caso, ad esempio, del secondo Dialogue); altre volte l'immagine ha funzione di contrasto: a una realtà insoddisfacente si contrappone la perfezione delle chimere immaginarie (si pensi a La Nouvelle Héloïe). In Jean-Jacques Rousseau et le discours sur la peinture (pp. 237-270) René DEMORIS mostra gli aspetti che avvicinano alcune analisi contenute nel primo Discours alle Réflexions di La Font de Saint-Yenne pubblicate anonime nel 1747 e mette in luce successivamente il rapporto tra il pensiero di Rousseau e alcuni testi settecenteschi sulla pittura. Rousseau et l'art du paysage (pp. 271-300) di Madeleine PINAULT SORENSEN si sofferma sulla visione della natura nei testi del ginevrino, visione articolata su tre punti tra loro legati: la formazione artistica di Rousseau, l'influenza dei testi letterari e l'apporto delle opere d' arte. Elisabeth LAVEZZI nel suo intervento dal titolo Rousseau et les discours sur la gravure (pp. 313-334) analizza le tesi di Rousseau sulle stampe mettendole a confronto con opere specializzate nel settore e appartenenti a un periodo che va dalla metà del ' 600 alla metà del ' 700 . Nella lettera 17 della quarta parte de La Nouvelle Héloïse l'immagine descritta da-Rousseau ricorda a tratti la sensibilità del pennello di Poussin, come mostra lo studio di Maria LEONE dal titolo Face à ce qui se dérobe: Rousseau avec Poussin. La Nouvelle Héloïse, quatrième partie, lettre 17 (pp. 411-426). Completano questa sezione un altro studio sull' estampe di Philip RoBinson dal titolo Rousseau et l'estampe: mieux qu 'un art visuel (pp. 301-312), l'analisi di Huguette KRIEF dedicata a «Le pinceau d'un Le Brun» ou l'art pictural du second Discours de Rousseau (pp. 335-356), l'intervento di Pascal GRIENER che si occupa di Gravelot au service de Rousseau et Voltaire: deux visions opposées de 
l'illustration (pp. 377- 410) e un ultimo articolo di Julia DOUTH WAITE dal titolo Visions du temps passé: Rousseau, Chardin et Greuze (pp. 427-456).

Una nuova sezione del volume, la quarta, dal titolo «Julie et Emile», si apre con le considerazioni di Philip STEWART sulle diverse forme e $i$ diversi significati dell'informazione visuelle che Rousseau ci offre a proposito di Julie (Le portrait de Julie, pp. 459-469), soffermandosi in particolare sul ritratto, vero talismano il cui potere sfugge alla sagacità di Wolmar. Si occupano ancora del romanzo i due studi successivi, uno di Ann M.THTORNTON dedicato a Rousseau graveur: une collection d'eaux fortes et d'états pour la Julie (pp. 471-510), l'altro intitolato Autour des sujets d'estampes de La Nouvelle Héloïse: estampes dramatiques et tableaux romanesques (pp. 511-528) opera di Catherine RAMOND. La sezione si conclude con un saggio di Isabelle MICHEL (Les illustrations de /'Emile au XVIIIe siècle: questions d'iconographie (pp. 529-563): l'autrice parte dalle stampe per l'edizione del 1762 presso Duchesne per passare poi a considerare gli illustratori di fine secolo, Moreau le Jeune, Cochin e Marillier. Risulta chiaro da questo studio come le illustrazioni del trattato sull'educazione riflettano la ricerca artistica e culturale dell'epoca che le ha prodotte, mentre il pensiero del ginevrino, di volta in volta reinterpretato nelle immagini, viene, nelle varie versioni, théâtralisé. Un'ultima breve sezione s'intitola «Mythes» e racchiude lo studio di Jacques BERCHTOLD, L'identification nourrie par l'iconographie: la cas de Diogène (pp. 567-582), originale saggio in cui l'autore mostra come la rappresentazione iconografica di Diogene sia all'origine di una forma di identificazione più volte ripresa da Rousseau. Il motivo si trova nei testi di Rousseau a partire dal 1738 e diviene ripetitivo dal 1753. L'assimilazione al protagonista dell'antichità diviene totale anche grazie ai continui avvicinamenti fatti dagli avversari del ginevrino (primo fra tutti Voltaire) tra la figura del ginevrino e quella di Diogene. Diogene con la lanterna diviene quindi per Jean-Jacques una figure-rempart, un modello consolatore lontano da una valutazione estetica e distaccato dalla tradizione iconografica. Le mythe de Rousseau chez Zurlauben et Laborde (pp. 583-617) è il titolo dell'articolo di Camilla MURGIA che chiude quest'ultima sezione, cui fa seguito una Annexe che racchiude un testo di Gauthier AMBRus dal titolo «Tais-toi Jean-Jacques». Variations sur une page de l'Emile (pp. 621-648). Conclude il volume l'Index onomastique ed un sentito necrologio di Jean Rousset redatto da Jean STAROBINSKI. 